\title{
SELECTION EFFECTS AND BINARY GALAXY VELOCITY DIFFERENCES
}

\section{Stephen E. Schneider, University of Massachusetts}

Edwin E. Salpeter, Cornell University

\section{Introduction}

Measurements of the velocity differences $(\Delta v$ 's) in pairs of galaxies from large statistical samples have often been used to estimate the average masses of binary galaxies. A basic prediction of these models is that the $\Delta v$ distribution ought to decline monotonically. However, some peculiar aspects of the kinematics have been uncovered, with an anomalous preference for $\Delta v \approx 72 \mathrm{~km} \mathrm{~s}^{-1}$ appearing to be present in the data.

We examine a large sample of binary galaxies with accurate redshift measurements and confirm that the distribution of $\Delta v$ 's appears to be non-monotonic with peaks at 0 and $\sim 72$ $\mathrm{kms}^{-1}$. We suggest that the non-zero peak results from the isolation criteria employed in defining samples of binaries and that it indicates there are two populations of binary orbits contributing to the observed $\Delta v$ distribution.

\section{The Observed Form of the $\Delta v$ Distribution}

For a complete sample of binary galaxies with velocity vectors oriented at random to the line of sight, the distribution of their observed $\Delta v$ 's should be a monotonically declining function of $\Delta v$. Even if there was a range of orbital velocities that actually were absent, the resultant distribution would only be flat over this range. Tifft (1980), however, found non-monotonic behavior in the $\Delta v$ distribution. Testing a hypothesis about possible nonmonotonic behavior against observational data is difficult if the hypothesis is made after the data is collected (Newman, Haynes, and Terzian 1989), but fortunately, both the quality and quantity of data on binary galaxies has improved greatly since Tifft (1980) developed his paradigm of the peculiar non-monotonic behavior.

Neutral hydrogen velocity-difference measurements of normal spiral galaxies made at 21 $\mathrm{cm}$ are now generally accurate enough to clearly distinguish between the posited $72 \mathrm{kms}^{-1}$ peak and the expected zero-velocity peak. Excluding the pairs from Tifft (1980), we have collected data on 102 isolated binaries that have accurate $21 \mathrm{~cm}$ velocities for both members in the literature (Schneider and Salpeter 1990). The distribution of $\Delta v$ 's is shown in Fig. 1 in the form of a frequency distribution $\mathrm{f}(\Delta v)$ (i.e., the value in the first bin is doubled to 
reflect its narrower range), using the same binning as suggested by Tifft (1980).

Fig. 1-Distribution of velocity differences among 102 binary galaxies measured at $21 \mathrm{~cm}$.

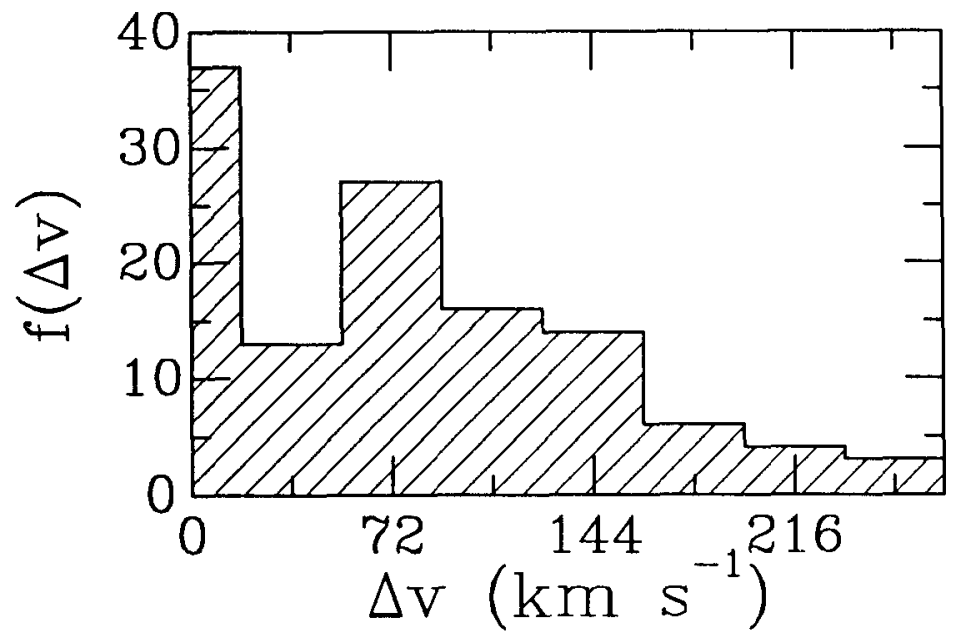

The distribution has fairly significant peaks in both the $0 \mathrm{~km} \mathrm{~s}^{-1}$ and $72 \mathrm{~km} \mathrm{~s}^{-1}$ bins; beyond $72 \mathrm{~km} \mathrm{~s}^{-1}$ the numbers decline smoothly. Other largely independent samples and measurements of binaries and groups also show a peak in the $72 \mathrm{~km} \mathrm{~s}^{-1} \mathrm{bin}$. Tifft (1980) also found peaks at higher multiples of $72 \mathrm{~km} \mathrm{~s}^{-1}$, but the evidence for any individual highervelocity peaks has never been very strong. Newman, Haynes, and Terzian (1989) have shown that any freedom in the choice of the zero point or bin size can produce apparently significant but spurious periodic peaks. Similar problems with the statistics seem likely to arise if special selection criteria are applied to the data after it is collected; any resultant enhancements produced in the appearance of periodicity become uninterpretable. We do not comment further on the analysis papers claiming to find this periodicity (Tifft and Cocke 1989 and references therein) except to note that the statistical significance of these works is difficult to evaluate because of sample overlap, differing selection criteria, and differing binning intervals.

\section{Effects of Incompleteness}

The binaries in our sample were selected on the basis of there being no likely massive companions within a few times the separation of the pair. This selection criterion should eliminate most groups with three or more comparably massive members, but the result is a bias in the final sample: pairs with large angular separations (and large projected linear separations) tend to be excluded. The binary samples are therefore incomplete. Of the pairs in our sample, $90 \%$ have projected separations $<136 \mathrm{~h}^{-1} \mathrm{kpc}$. This cutoff has some interesting implications for the form of the $\Delta v$ distribution. 
The projected-separation cutoff will eliminate preferentially those pairs lying most nearly perpendicular to the line of sight. A selection procedure will influence $\mathrm{f}(\Delta v)$ when the apparent separation and velocity of pairs are interdependent. For circular orbits, the largest projected $\Delta v^{\prime}$ occur when the projected separations are largest, while for eccentric orbits, the smaller $\Delta v$ 's occur near apocenter, so a projected-separation cutoff may influence $f(\Delta v)$.

When a projected-separation cutoff is applied to orbits of even small eccentricity a nonmonotonic distribution can result. In Fig. 2, the effect of different projected separation cutoffs is shown for circular orbits and for orbits with an eccentricity of 0.33 . These are analytic models, numerically integrated, in which we follow a Keplerian orbit, consider all possible orientations, and find the resultant projected separation and the line-of-sight velocity at equal time intervals around the entire orbit. For both eccentricities we show the complete sample, and then we apply more restrictive projected separation cutoffs of $\frac{1}{4}, \frac{1}{8}$, and $\frac{1}{16}$ of the apocenter separation; the velocities are scaled relative to the pericenter velocity of the orbit.
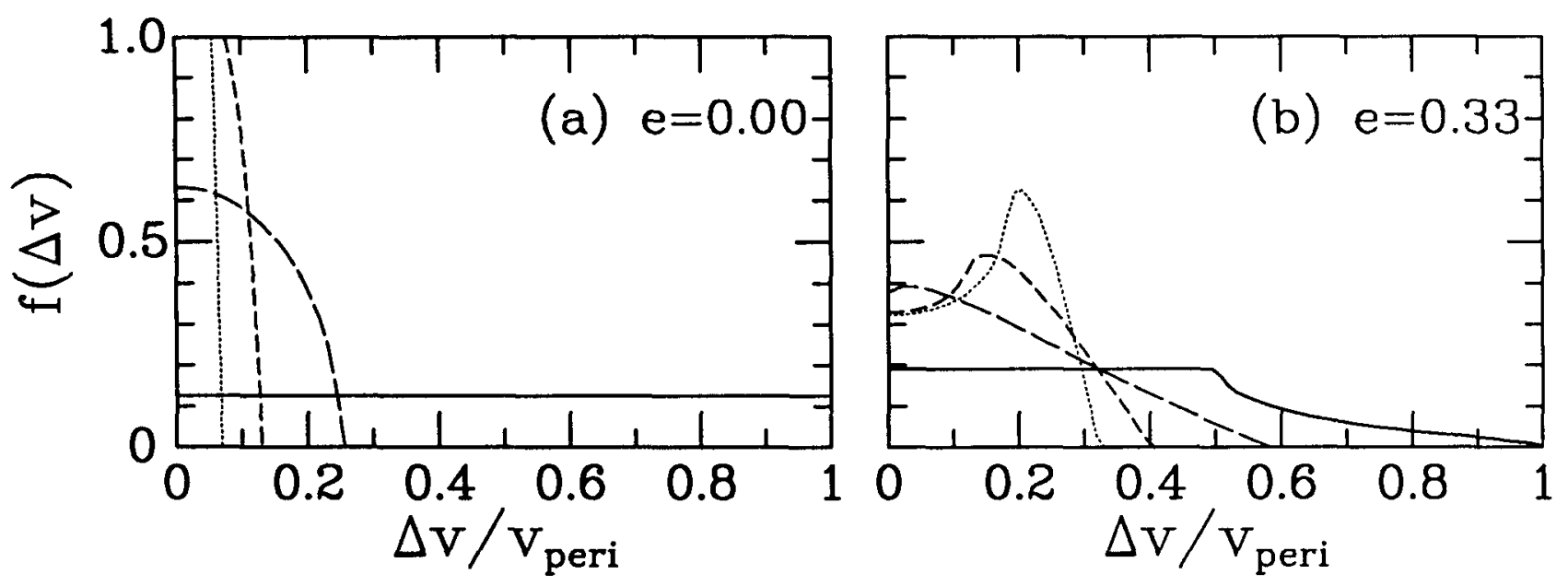

Fig. 2-Distribution of expected velocity differences for (a) circilar and (b) eccentric orbits, using a complete sample (solid line) and then applying successively narrower projected-separation cutoffs at $\frac{1}{4}$ (long dash), $\frac{1}{8}$ (short dash), and $\frac{1}{16}$ (dotted) of the apocenter separation.

For non-circular orbits in general, $\mathrm{f}(\Delta v)$ peaks toward $\Delta v=0$ in a complete sample because the binaries spend more time at larger separations orbiting more slowly. The application of smaller cutoff radii makes $\mathrm{f}(\Delta v)$ more and more distinctly non-monotonic as the lower velocities are removed, and this could quite reasonably contribute to a peak near $72 \mathrm{~km} \mathrm{~s}^{-1}$ since the peak occurs at some fraction of the typical orbital velocity. 
Of course, in a realistic model, a wide range of orbital parameters would be likely, which might "wash out" the effects seen for this simple Keplerian analysis. We therefore constructed a more detailed model in which we traced orbital parameters for a full population of pairs from the time of their formation, when they would have been expanding with the Hubble flow. We allowed a Gaussian distribution of angular momenta and then applied projected separation cutoffs to the sample at the present day. Interestingly, the only natural velocity scale in the model is the escape velocity of the pair on a zero-energy trajectory. Solving the energy-balance equation, this velocity is $v_{\text {esc }}=\left(\frac{4}{3} G M / t_{H}\right)^{1 / 3} \approx 72 \mathrm{~km} \mathrm{~s}^{-1}$ for $M \approx 10^{12} M_{\odot}$.

The pairs still expanding at present are the most widely separated and when a projected separation cutoff is introduced, they contribute only slightly to $f(\Delta v)$. On the other hand, the pairs that had initially small separations will presently be merging, and the major axes of their orbits will be made smaller still by dynamical friction. The intermediate "converging" pairs, which are falling back together, but which have not yet collided, prove to have the most interesting properties with respect to the projected-separation criterion. In Fig. 3 , we show the effect of selection criteria on the population of converging pairs, with projected separation cutoffs relative to the escape separation as in Fig. 2. The non-zero peak in $\mathrm{f}(\Delta v)$ occurs at a velocity similar to $72 \mathrm{~km} \mathrm{~s}^{-1}$ for the known projected separation cutoff when the average pair mass is $\sim 3 \times 10^{11} \mathrm{M}_{\odot}$.

Fig. 3-Distribution of expected velocity differences for a modelled range of orbital parameters, using various projected separation cutoffs (relative to the escape-velocity separation) as in Fig. 2.

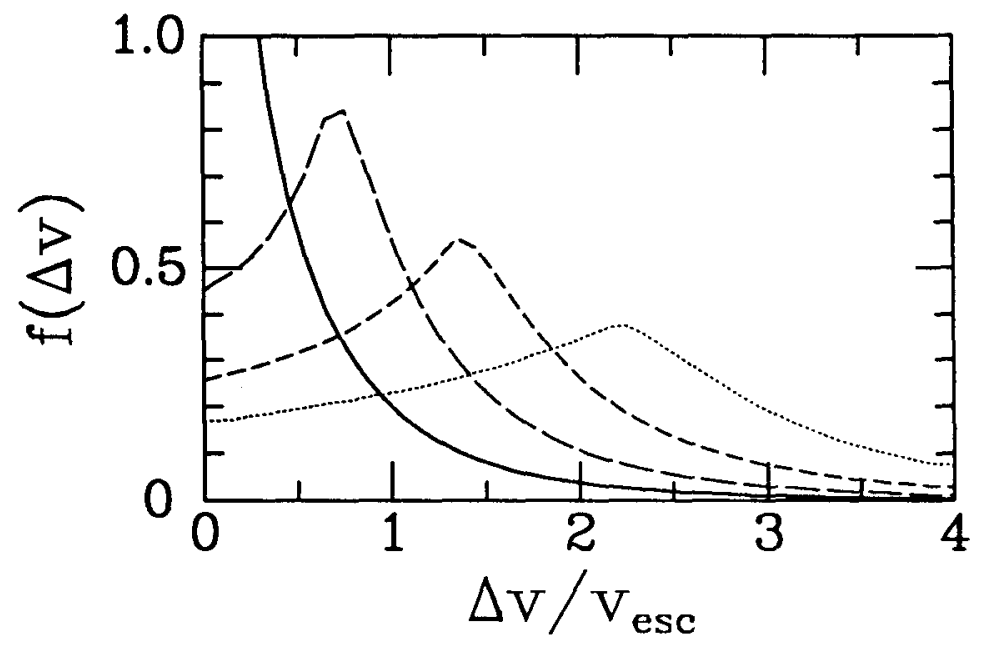

The peak around $\Delta v=0$ in the observed distribution (Fig. 1) could be produced by the population of merging pairs, which would be more completely sampled because of their smaller major axes. A reasonable facsimile of this peak could be produced, for example, by 
completely sampled $e=0.7-0.8$ orbits with typical $200 \mathrm{~km} \mathrm{~s}^{-1}$ rotation speeds at pericenter.

\section{Discussion}

In summary, we see evidence of non-monotonic behavior in $\mathrm{f}(\Delta v)$ for $\Delta v<100 \mathrm{~km} \mathrm{~s}^{-1}$. We have constructed a fairly realistic model of binary orbits, and we find that a peak occurs in the population of "converging" pairs at a velocity of the order of magnitude of $\left(G M H_{o}\right)^{1 / 3} \approx$ $72 \mathrm{~km} \mathrm{~s}^{-1}$. We further speculate that the peak at $0 \mathrm{~km} \mathrm{~s}^{-1}$ is produced merging pairs.

We can make some predictions based on our two population model. For uniform samples of isolated pairs, we expect that the non-zero peak will move to larger values of $\Delta v$ as the cutoff radius is decreased or the pair mass (based on absolute magnitudes, for example) increases. For current samples this point is difficult to test because of another bias present in the data-the isolation criteria also tend to select more massive pairs at larger projected separations. This bias may actually help keep the non-zero peak in $\mathrm{f}(\Delta v)$ from being smoothed out despite a range of contributing binary galaxy masses.

We suspect, therefore, that previous dynamical estimates of binary galaxy masses are likely to be in error. Even if our two-population model does not prove correct, it seems clear that selection effects must significantly limit the range of selected orbital orientations in binary samples, and these effects need to be better understood. If our model is correct, only the merging pair population has been (approximately) completely sampled, and the characteristic width of its $\mathrm{f}(\Delta v)$ distribution is substantially narrower than has been previously suspected; as a result these pairs' masses have probably been overestimated. We conclude, though, with a more positive message: if there is a non-zero peak in $\mathrm{f}(\Delta v)$, it may ultimately provide a much better tool for estimating not only the masses of converging binaries but for deciphering the origin and evolution of binary orbits as well.

\section{References}

Newman, W. I., Haynes, M. P., and Terzian, Y. 1989, Ap. J., 344, 111.

Schneider, S. E., and Salpeter, E. E. (1990) in preparation.

Tifft, W. G. 1980, Ap. J., 236, 70.

Tifft, W. G., and Cocke, W. J. 1989, Ap. J., 336, 128. 


\section{DISCUSSION}

Miller: How bad will virial mass estimates be? Is it a 108 effect, a factor of two, or what?

Schneider: The mean velocity differences will tend to be overestimated, but the separations underestimated. The net effect will tend to be overestimated masses, but based on my simple models, probably only by factors like two for typical cutoff separations. 